\title{
Terahertz and mm-wave applications at ENEA-Frascati
}

\author{
G. P. Gallerano, S. Ceccuzzi, A. Doria, E. Giovenale, G. L. Ravera, M. Zerbini, \\ G. Galatola-Teka ${ }^{2}$ \\ ${ }^{1}$ ENEA - Fusion Physics Division - Radiation Sources, Antennas and Diagnostics Laboratory \\ Via E. Fermi 45, 00044 Frascati - Italy, gianpiero.gallerano@enea.it \\ ${ }^{2}$ ENEA Phd Student
}

Frequencies in the $\mathrm{THz}$ region are usually considered as too high for electronic devices, due to the technological limits in reducing the size of components as frequency increases, and too low for photonic devices, since the corresponding photon energy is comparable to those of thermal excitations at room temperature. A variety of applications of $\mathrm{THz}$ radiation are being developed worldwide. The majority of applications exploits the spectral fingerprints of the chemical species contained in the material under investigation and takes advantage from the fact that $\mathrm{THz}$ radiation can penetrate through plastic, cardboard and clothes, without causing the hazards of ionizing radiation (X-ray) and suffering at the same time less scattering than near-infrared radiation.

This wide variety of applications requires an equally wide range of sources and radiation characteristics. This is the reason why two Free Electron Lasers (FELs) have been designed, developed and realised at the ENEA Frascati Research Centre [1]. Thanks to their intrinsic generation mechanism they have the peculiar advantage of being tunable sources.

The ENEA Compact FEL is based on a microtron electron accelerator capable of generating a relativistic beam at an energy of $2.3 \mathrm{MeV}$ with a current of $300 \mathrm{~mA}$, in $4 \mu \mathrm{s}$ pulses. A magnetic undulator, designed and realised by ENEA, made of 8 permanent magnets ( $\mathrm{SmCo}$ ) periods, provides the generation of radiation in the range $100-150 \mathrm{GHz}$ with a peak power of $1.5 \mathrm{~kW}$ over the macropulse. Due to the RF nature of the accelerator, a substructure inside the pulse generates a train of micropulses of about 60 ps duration, equally spaced by 330 ps, with a peak radiation power of about $10 \mathrm{~kW}$. The average $\mathrm{CW}$ power can be varied according to the repetition rate of the $\mathrm{RF}$ system driving the accelerator providing about $15 \mathrm{~mW}$ at $2.5 \mathrm{~Hz}$ (Fig. 1).

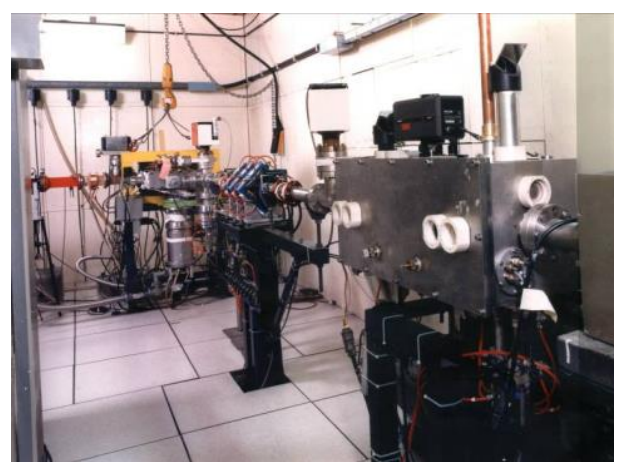

Fig. 1. The ENEA Compact FEL
The second ENEA FEL source is named FEL-CATS (Compact Advanced THz Source) and it is a unique device in the FEL panorama. It is a compact source based on a Linac type accelerator (2.3 $\mathrm{MeV}$ of energy and $250 \mathrm{~mA}$ of average current over $5 \mu \mathrm{s}$ ). It includes an additional RF device (called PMD), placed downstream the Linac, capable of manipulating the electrons in the longitudinal phasespace in such a way to create an adequate level of order in the bunch, to enhance the electromagnetic generation mechanism. This unique source, due to the presence of the PMD, does not make use of an optical resonator, thus extending its tuning capability over almost one octave. In fact, it generates radiation from $400 \mu \mathrm{m}(0.7 \mathrm{THz})$ to $700 \mu \mathrm{m}(0.4 \mathrm{THz})$ with a power level of several $\mathrm{kW}$ over the microsecond pulse duration (see Fig. 2).

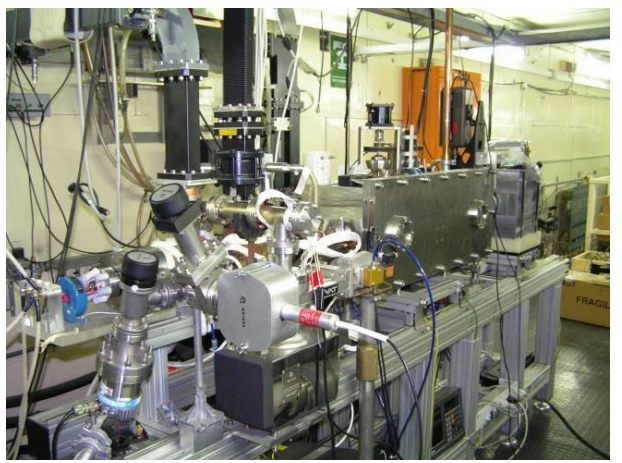

Fig. 2. The ENEA FEL-CATS source

Beside FEL sources, our laboratory has been equipped with an extensive assortment of other coherent sources, both lasers and electron devices as a part of the Center of Excellence for Terahertz Radiation and Applications (CETRA). A Far InfraRed (FIR) gas laser based on a $\mathrm{CO}_{2}$ laser, pumping a gas cell, is available as well as a Ti:Sa laser oscillator that generates a train ultra-short pulses (about $130 \mathrm{fs}$ ) in the Near InfraRed (NIR) with an average CW power in excess of $1 \mathrm{~W}$. Other compact, portable, solid state sources are available in the $\mathrm{THz}$ laboratory to cover the low frequency spectrum: two YIG oscillators that emit in the ranges 8-20 and 20-40 GHz respectively, with an average $\mathrm{CW}$ power of $5-10 \mathrm{~mW}$; an IMPATT diode at $97 \mathrm{GHz}$ with about $80 \mathrm{~mW}$ of output power and a second IMPATT diode emitting at $140 \mathrm{GHz}$ with about $20 \mathrm{~mW}$ of power. Finally a frequency multiplier in the range $220-330 \mathrm{GHz}$ is also available to test high frequency components. 
The CETRA Laboratory has realised an extensive variety of applications in different scientific and technological fields, like living plants biology, human biology, solid state physics and cultural heritage protection. The most notable activity was the pioneering THz BRIDGE project $(2001$ - 2004), a European project that opened the way to the study of the interaction of $\mathrm{THz}$ radiation with biological systems following a streamline of increasing complexity, from bio-molecules to living cells and tissues. At present the utilization capability of the laboratory resources takes place mainly in the context of scientific collaborations funded through national or international projects.

Recent applications in the biomedical field, are carried out in the frame of the GREAM project [2-3], financed by the Italian Ministry of Defence, and was realised in collaboration with the Scientific Department of Army Medical Center of Rome, and the Universities of Rome "La Sapienza", "Tor Vergata" and "Roma Tre". It focused on the study of potential genotoxic effects induced on different kind of human fibroblasts, after irradiation with the Compact FEL source, in the $100-150 \mathrm{GHz}$ frequency range, and with the YIG source at $25 \mathrm{GHz}$. In order to correctly characterize the sample exposition, a preliminary study on the Specific Absorption Rate (SAR) has been performed, also by means of simulations, as reported in Fig. 3.

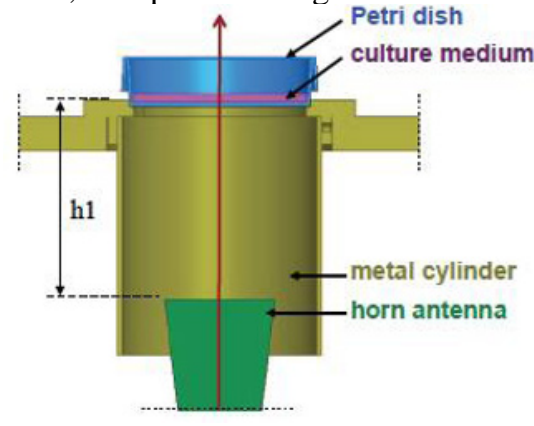

a)

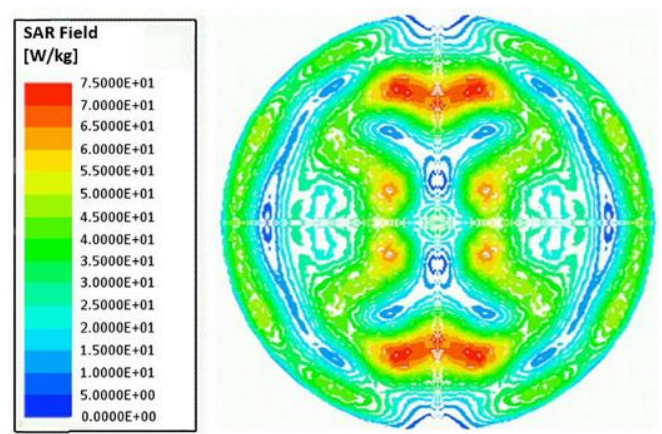

b)

Fig. 3. (a) Detail of the setup showing the position of the petri dish; (b) SAR distribution on the cell layer at the bottom of the petri dish

In the field of Cultural Heritage preservation, a specific project, THz-ARTE, was carried out in the frame of a bilateral collaboration between Italy and Japan. For this project the CETRA Laboratory has realized an innovative imaging system [4] for the analysis of artwork samples, which were prepared by the NICT Institute in Tokio and by the CNR-IFAC Institute in Florence (see Fig. 4).

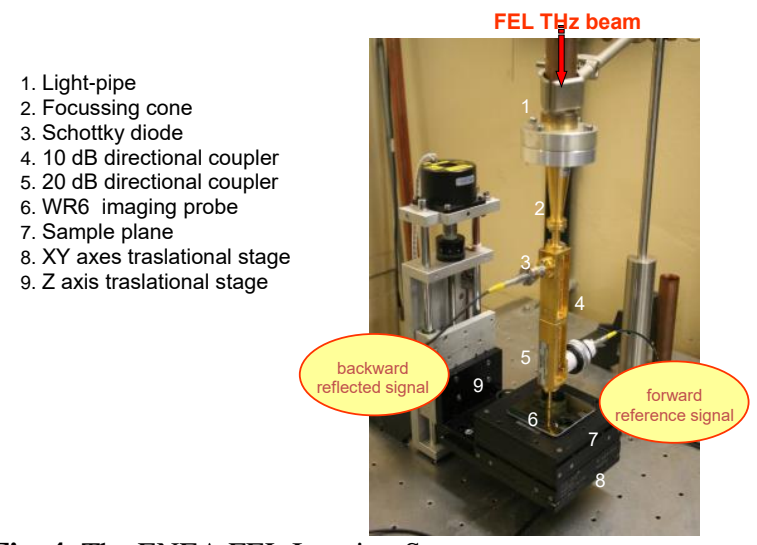

Fig. 4. The ENEA FEL Imaging System

A second reflective imaging system, employing the $97 \mathrm{GHz}$ IMPATT source, has been realised to be suitable for "on field" operation. It exploits the penetrating features of the MM wave radiation together with a phase sensitive detection technique. This imaging system has been successfully operated on real ancient paintings (see Fig. 5).

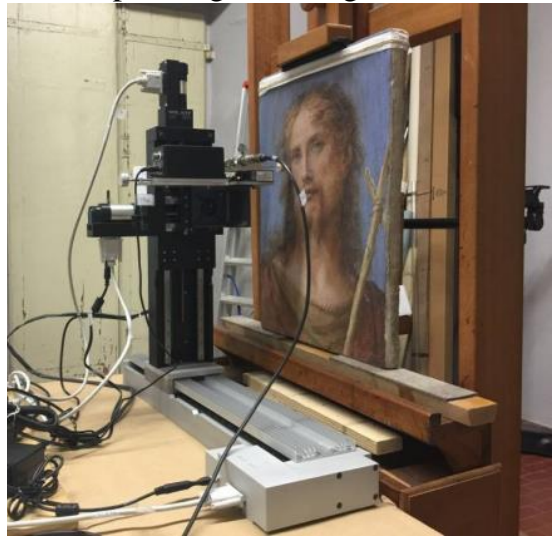

Fig. 5. Portable ENEA Imaging system applied to a "Fresco" painting on the "tavella"-St. John Baptist - by Alessandro Gherardini (1655-1726)

\section{References}

1. Biedron, S., Gallerano, G. P., et al. Compact, High Power Electron Beam based Terahertz Sources // Proc. Of the IEEE 2007. V. 95, No. 8 P. 1666-1678.

2. Franchini, V., et al. Study of the Effects of $0.15 \mathrm{Te}-$ rahertz Radiation on Genome Integrity of Adult Fibroblasts // Environmental and Molecular Mutagenesis 2018 V. 59, P. 476-487.

3. Franchini, V., et al. Biological Effects of 25 to 150 $\mathrm{GHz}$ Radiation after in Vitro Exposure of Human Fibroblasts: a Comparison of Experimental Results // J Infrared Milli Terahz Waves 2018, V. 39 P. 899-911.

4. Gallerano, G. P., Doria, A., Germini, M., Giovenale, E., Messina, G., Spassovsky, I. Phase-Sensitive Reflective Imaging Device in the mm-wave and Terahertz Regions // J Infrared Milli Terahz Waves 2009. V. 30 P. 1351-1361.

5. Doria, A. Gallerano, G. P., Giovenale, E., Casini, A., Cucci, C., Picollo, M., Poggesi, M., Stefani, L., Fukunaga, K., Tamassia, M., Vis-NIR Hyper-spectral and Terahertz Imaging Investigations on a Fresco Painting on "Tavella" by Alessandro Gherardini // J Infrared Milli Terahz Waves 2017, V. 38, P. 390-402 